\title{
Incidence of Pasteurellosis in a Pigeon (Columba livia) - A Case Report
}

\author{
S. Ramesh ${ }^{1}$, C. Soundararajan ${ }^{2}$, K. Manimaran ${ }^{4}$, S. Subapriya ${ }^{3 *}$ and R. Sokkalingam ${ }^{5}$ \\ ${ }^{1}$ Centralised Instrumentation Laboratory, ${ }^{2}$ Department of Veterinary Parasitology, \\ ${ }^{3}$ Centralised Clinical Laboratory, Madras Veterinary College, Chennai-07, India \\ ${ }^{4}$ Department of Veterinary Public Health and Epidemiology, Veterinary College and Research \\ Institute, Thanjavur, India \\ ${ }^{5}$ Society for the Prevention of Cruelty to Animals (SPCA), Vepery, Chennai-07 \\ *Corresponding author
}

\section{A B S T R A C T}

\section{Keywords}

Pigeon, Columba livia, Pasteurellosis

Article Info

Accepted:

30 November 2018

Available Online:

10 December 2018

\begin{abstract}
A domestic pigeon which was brought to SPCA, Chennai by a pet owner having 50 pigeons was referred for disease diagnosis with the history of sudden mortality and respiratory distress. The remaining ailing birds were inspected and were found to be lethargic with respiratory distress. Necropsy examination revealed grayish white foci scattered on the liver parenchyma. Liver impression smears and heart blood smears were suggestive of Pasteurella spp. Cultural examination of heart blood confirmed $P$. multocida. Antibiotic sensitivity assay revealed high sensitivity to enrofloxacin. Based on the laboratory findings, the other birds were treated with enrofloxacin for five days. The birds became active and normal in feeding habits after 5 days of treatment.
\end{abstract}

\section{Introduction}

Avian pasteurellosis, caused by Pasteurella multocida is an infectious septicemic disease affecting both domestic as well as wild birds. The disease was reported in several caged birds (Mehrothra et al., 2000 and Sawada et al., 1999). In free ranging birds, P. multocida has been reported as a causative agent in over 100 species and almost all species are susceptible under certain circumstances (Pedersen et al., 2003). The disease is transmitted either by inhalation of a bacterialadened aerosol or by consumption of contaminated water or food (Botzler, 1991). It causes respiratory disease in more than 180 species of wild birds (Samuel et al., 2005). In acute outbreaks, wild waterbirds in good body condition may be found dead with few or no clinical signs within hours of exposure (Friend, 1999; Samuel et al., 2007; Ladds, 2009). Clinical signs, when seen, are generally non specific and may include lethargy, nasal discharge and a range of neurological signs (e.g. erratic swimming and flight, convulsions). Scavenger bird species typically die 1-2 weeks after infection, often distant from the geographical site of exposure (Friend 1999). The present paper reports an incidence of Pasteurellosis in pigeon and effective treatment with enrofloxacin. 


\section{Materials and Methods}

A domestic pigeon brought to Society for the Prevention of Cruelty to Animals (SPCA) Hospital, Chennai by a pet owner at Chennai was referred for disease diagnosis. A total number of 50 pigeons maintained in cages at the terrace were reported to have signs of respiratory distress with history of sudden mortality in four birds. The ailing birds were inspected and a detailed necropsy was performed on the dead bird. Gross lesions were recorded. The heart blood smears and liver impression smears collected were stained by Leishman's stain and subjected to microscopic examination.

Heart blood swab was collected and directly inoculated on Brain Heart Infusion (BHI) agar plates and incubated at $37^{\circ} \mathrm{C}$ overnight. Further, isolated colonies were subjected to biochemical tests like catalase, oxidase, indole, methyl red, Voges-Proskauer, citrate, and urease tests. All the samples were subjected to PCR targeting the kmt 1 gene and capsular typing. In addition antibiotic sensitivity assay was also performed using heart blood swab. Based on the laboratory findings, the ailing birds were treated with enrofloxacin @ $5 \mathrm{mg} / \mathrm{kg}$ body weight for five days. The birds became active and normal in feeding habits after 5 days of treatment.

\section{Results and Discussion}

The ailing birds in the cages were found to be lethargic (Fig. 1) and exhibited respiratory distress with mucoid nasal discharges. The droppings were brownish and watery in consistency.

External examination of the carcass of the dead bird revealed no abnormalities except mucoid discharges from nostrils. Gross examination revealed areas of congestion and consolidation in lungs while liver revealed few grayish foci scattered on the parenchyma of liver (Fig. 2). On incision, these grayish foci were seen extending deep into the parenchyma. Other organs viz kidney, spleen and intestine revealed only congestion. The intestinal contents were brownish in colour and watery in consistency which on microscopic examination revealed no endoparasites. Heart blood smears and liver impression smears revealed the presence of bipolar organisms suggestive of Pasteurella spp. (Fig. 3). Inoculation on BHI Agar revealed characteristic colonies which were small, white, pasty, dew drop like, circular and translucent, confirmative of P.mutocida (Fig. 4).

Isolated colonies were subjected to Gram staining and biochemical tests like catalase, oxidase, indole, methyl red, Voges-Proskauer, citrate, and urease tests. Colonies having characteristics of dewdrop and non-hemolytic appearance on blood agar were used further for biochemical testing. Upon Gram staining, the appearance of gram-negative coccobacilli was noticed. Isolated colonies showed no growth on MacConkey agar and tests like catalase, oxidase, and indole were positive (Fig. 5).

Methyl red, Voges-Proskauer, citrate and urease tests were negative for the isolates.All the samples subjected to PCR targeting the kmt1 gene showed a band at 464 bp (Fig. 6) and capsular typing results amplified at 1044bp (Fig. 7) and the results revealed that all the isolates were of Pasteurella multocida Type-A.

Antibiotic sensitivity assay revealed high sensitivity to enrofloxacin, intermediate sensitivity to cephalexin and resistance to gentamicin, tetracycline, streptomycin and ampicillin. The ailing birds became active and normal in feeding habits after 5 days of treatment with enrofloxacin. 
Fig.1 Pigeon-pasteurellosis - lethargic

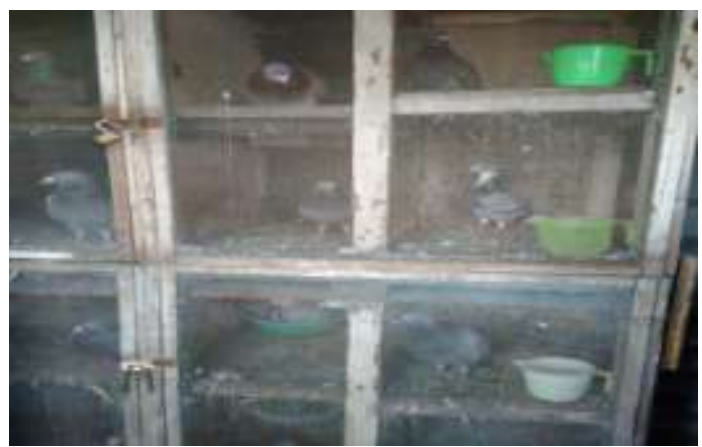

Fig.2 Pigeon-pasteurellosis - liver - greyish foci

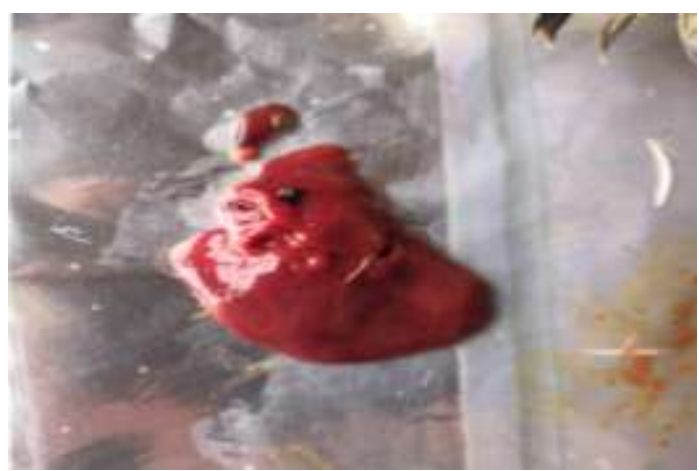

Fig.3 Pigeon- Heart blood smear - Giemsa stain-Bipolar organisms

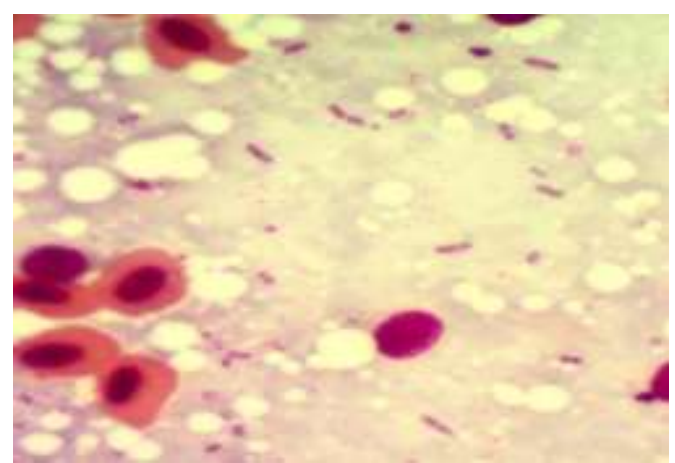

Fig.4 Pigeon-BHI Agar- Dew drop colonies -Pasteurella multocida

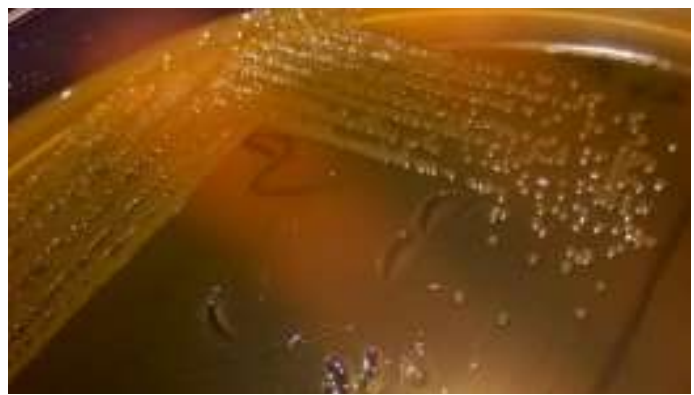


Fig.5 Biochemical test- Indole, Oxidase, Catalase- Positive

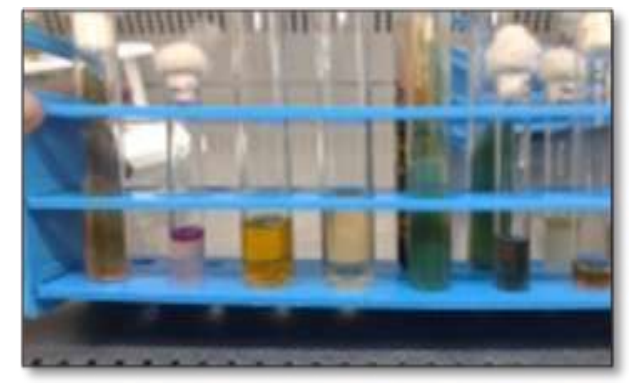

Fig.6 Pasteurella multocida kmt gene specific PCR

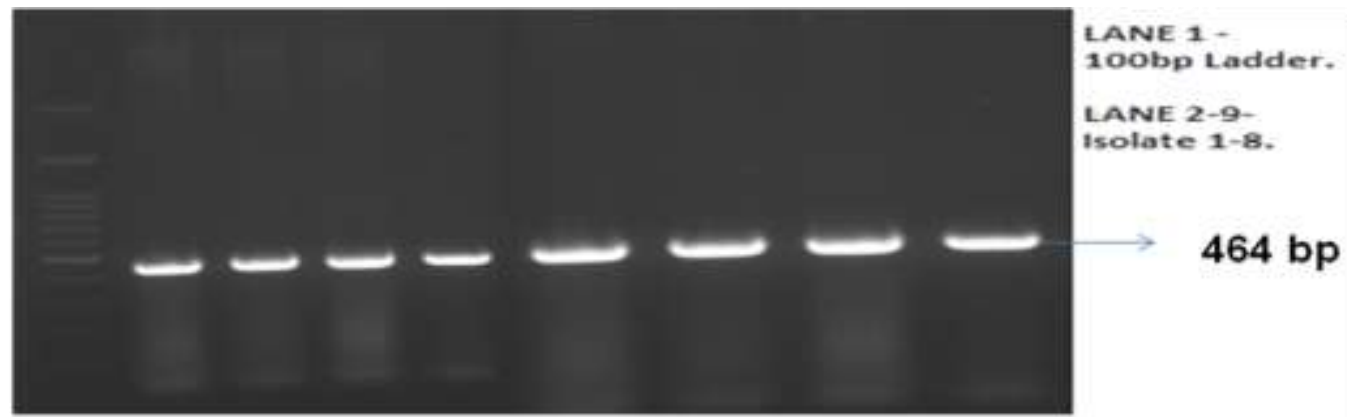

1.5\% agarose gel electrophoresis of $\mathrm{kmt}$ gene amplicons at 464 bp

Fig.7 Pasteurella multocida capsular type-A PCR

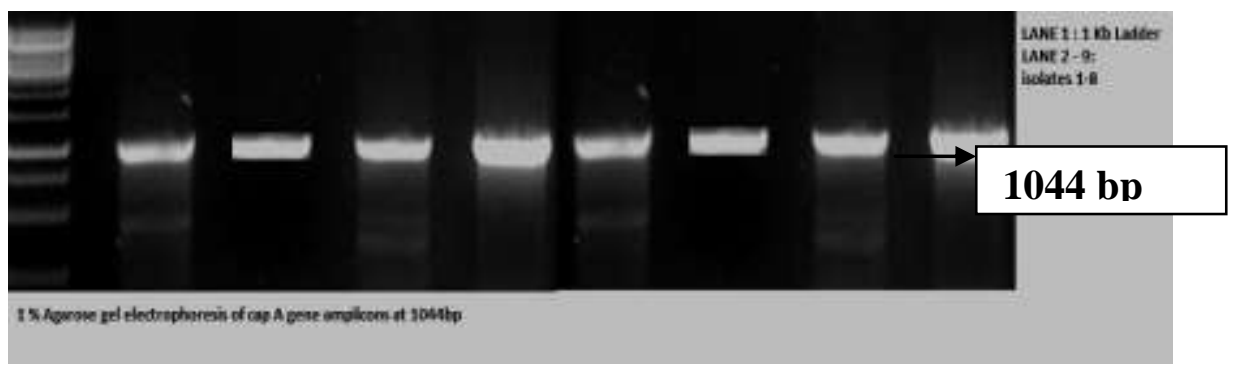

Jayathangaraj et al., (2011) reported an incidence of avian pasteurellosis in pariah kites. They also observed numerous grayish foci on the liver extending deep into the parenchyma and congestion in other organs as observed in the present study. Similar findings were also recorded by Sathasivam et al., (2009) in White goose infected with Pasteurellosis. Jayathangaraj et al., (2011) also found that treatment with enrofloxacin @ $5 \mathrm{mg} / \mathrm{kg}$ body weight were effective in Pasteurella infected Geese.
Pasteurella organisms are opportunistic pathogens, commonly found in the respiratory tract of animals and birds without causing clinical disease. During stress i.e. overcrowding, change in weather, starvation or poor sanitation, the organisms cause the disease (Jayathangaraj et al., (2011). In the present case poor managemental conditions were found to be the cause of the disease. Hence the owner was advised to follow proper disinfection measures after disposal of the carcasses which may act as prime source 
of further infection to other birds and to provide clean and fresh water and feed to avoid reinfection.

\section{References}

Botzler, R.G. 1991. Epizootiology of avian cholera in wildfowl. Journal of Wildlife diseases 27(3): 367-395

Jayathangaraj, M.G., Ramesh, S., Basith, S.A. and Purushothaman, V. Incidence of Avian Pasteurella in Pariah Kites (Milvus migrans). 2011. Indian. J. Anim. Hlth., 50 (1): 44-45

Pedersen, K., Dietz, H.H., Jørgensen, J. C., Christensen, T.K., Bregnballe, $\mathrm{T}$ and
Andersen, T.H. 2003. Pasteurella multocida from outbreaks of Avian Cholera in wild and captive birds in Denmark. Journal of Wildlife Diseases, 39(4): 808-816

Samuel, M.D., Goldberg, D.R., Shadduck, D.J., Price, J.I. and Cooch, E.G., 1997. Pasteurella multocida serotype 1 from a lesser snow goose: Evidence of a carrier state. Journal of Wildlife Diseases. 33: 332-335

Sathasivam, S, Khan, P.N., Senthil Kumar, K., Mytheen, F., Ramesh, S and Jayathangaraj, $\quad$ M.G. 2009. Pasteurellosis in White Goose. Zoos' Print. 24(7).

\section{How to cite this article:}

Ramesh, S., C. Soundararajan, K. Manimaran, S. Subapriya and Sokkalingam, R. 2018. Incidence of Pasteurellosis in a Pigeon (Columba livia) - A Case Report. Int.J.Curr.Microbiol.App.Sci. 7(12): 3693-3697. doi: https://doi.org/10.20546/ijcmas.2018.712.419 